\title{
Impact of an extra gauge interaction on naturalness of supersymmetry
}

\author{
Marcin Badziak $^{a}$ and Keisuke Harigaya ${ }^{b, c}$ \\ ${ }^{a}$ Institute of Theoretical Physics, Faculty of Physics, University of Warsaw, \\ ul. Pasteura 5, PL-02-093 Warsaw, Poland \\ ${ }^{b}$ Department of Physics, University of California, \\ Berkeley, California 94720, U.S.A. \\ ${ }^{c}$ Theoretical Physics Group, Lawrence Berkeley National Laboratory, \\ Berkeley, California 94720, U.S.A. \\ E-mail: mbadziak@fuw.edu.pl, keisukeharigaya@berkeley.edu
}

ABSTRACT: It is pointed out that in supersymmetric models with a new gauge symmetry under which the Higgs is charged, the fine-tuning of the electroweak symmetry breaking is relaxed due to suppression of the top Yukawa coupling at higher scales by a new large gauge coupling. We calculate the fine-tuning in an explicit model and find that the lower bounds on stops and gluino masses from the naturalness criterion are increased by several hundred GeV in comparison to the Minimal Supersymmetric Standard Model (MSSM). The fine-tuning is improved by one to two orders of magnitude as compared to the MSSM, as a consequence of both the suppression of the top Yukawa coupling and the additional tree-level contribution to the Higgs mass allowing for much lighter stops.

KEYwords: Beyond Standard Model, Supersymmetric Standard Model

ArXiv EPrint: 1806.07900 


\section{Contents}

1 Introduction 1

2 Supersymmetry with an extra gauge symmetry 2

2.1 Suppression of the top Yukawa by an extra gauge interaction 2

2.2 A model with an extra $\mathrm{SU}(2)$ symmetry 3

3 Naturalness of electroweak symmetry breaking 5

$\begin{array}{lll}4 & \text { Summary and discussion } & 10\end{array}$

\section{Introduction}

Supersymmetry (SUSY) provides one of the best solutions to the hierarchy problem of the Standard Model (SM) [1-4]. However, the minimal version of the realization of this idea, the Minimal Supersymmetric Standard Model (MSSM), has been put under a strong pressure by the LHC results. The primary problem of the MSSM is the fact that explaining the $125 \mathrm{GeV}$ Higgs mass [5] requires multi-TeV stops which implies large fine-tuning of the electroweak (EW) scale, the so-called little hierarchy problem. This has motivated a great amount of work on various extensions of the MSSM in which new positive contributions to the Higgs mass are present which allow for the $125 \mathrm{GeV}$ Higgs mass without heavy stops. The most notable examples of such models are Next-To-Minimal Supersymmetric Standard Model (NMSSM) [6-14] and models with non-decoupling $D$-terms of some new gauge interactions under which the Higgs is charged [15-22]. However, even if the observed Higgs mass is obtained with light stops the fine-tuning is still present due to constraints on supersymmetric particles, especially on stops and gluino, from direct LHC searches [23, 24].

The fine-tuning is particularly large for models with high mediation scale of SUSY breaking, thus disfavouring many simple mechanisms of SUSY breaking such as gravity mediation. This motivated construction of new models with extremely low scale of SUSY breaking, see e.g. [25, 26], models with Dirac gauginos [27, 28] which do not have logarithmicly-enhanced contributions to the Higgs mass parameter, and SUSY models [2933 ] which suppress the fine-tuning and enhance the tree-level Higgs mass by invoking the Twin Higgs mechanism [34-36].

In the present paper, we point out that in a class of SUSY models with a new gauge symmetry under which the Higgs is charged, the top Yukawa coupling becomes small at high energy scales by the renormalization from the new gauge interaction. As a result, the logarithmically-enhanced quantum correction to the Higgs mass parameter is suppressed, making models with high mediation scale of SUSY breaking not less motivated from the naturalness perspective than models with a low mediation scale. This mechanism is generic 
as long as the new gauge coupling is large, which requires the new interaction to be asymptotically free to stay in the perturbative regime up to high scales. As a demonstraton of this idea, we quantify in detail naturalness of the EW symmetry breaking (EWSB) in a specific model with $\mathrm{SU}(2)_{X} \times \mathrm{SU}(2)_{W}$ gauge symmetry which is broken to the $\mathrm{SM} \mathrm{SU}(2)_{L}$ gauge group at a scale of around $10 \mathrm{TeV}$. We find that the fine-tuning may be relaxed by a factor of few as compared to the MSSM and particularly large improvement is found in the inverted sfermion mass hierarchy scenario in which the first two generations of sfermions are much heavier than the third one. Moreover, after the Higgs mass constraint is taken into account the improvement in the tuning, as compared to the MSSM, is one to two orders of magnitude.

The article is organized as follows. In section 2 we discuss how the suppression of the top Yukawa coupling is obtained and introduce a model in which this scenario can be realized. In section 3 we quantify the fine-tuning of the model and compare it to the MSSM. We reserve section 4 for the summary and concluding remarks.

\section{$2 \quad$ Supersymmetry with an extra gauge symmetry}

In this section we describe how in a model with an extra gauge symmetry the quantum correction to the Higgs mass parameter from stops and gluino is suppressed, and introduce an explicit example.

\subsection{Suppression of the top Yukawa by an extra gauge interaction}

In the MSSM, the soft mass of the Higgs $m_{H_{u}}^{2}$ receives a large quantum correction from those of stops $m_{Q_{3}}^{2}, m_{U_{3}}^{2}$ because of the large top Yukawa coupling $y_{t}$,

$$
\frac{\mathrm{d}}{\mathrm{d} \ln \mu_{R}} m_{H_{u}}^{2}=\frac{6 y_{t}^{2}}{16 \pi^{2}}\left(m_{Q_{3}}^{2}+m_{U_{3}}^{2}\right)+\cdots
$$

The effect is especially significant for a large mediation scale of SUSY breaking because of the large logarithmic enhancement, which leads to an excessive amount of the fine-tuning to obtain the EWSB scale.

We point out that this problem is relaxed in extensions of the MSSM such that the Higgs is charged under extra gauge symmetry, which have been extensively discussed becuase they alllow for the $125 \mathrm{GeV}$ Higgs mass with relatively small stop masses [15-22]. The running of the top Yukawa coupling is given by

$$
\frac{\mathrm{d}}{\operatorname{dln} \mu_{R}} y_{t}=\left(\gamma_{H_{u}}+\gamma_{Q_{3}}+\gamma_{\bar{U}_{3}}\right) y_{t},
$$

where $\gamma_{i}$ is the anomalous dimension of the field $i$. At one-loop level gauge interactions gives a negative contribution to anomalous dimensions, suppressing the top Yukawa coupling at high energy scales. As a result the quantum correction to $m_{H_{u}}^{2}$ is also suppressed.

We are interested in a model which remains perturbative up to a high energy scale and does not require a UV completion below the energy scale of gravity. In order to make the suppression effective by a large gauge coupling, the extra gauge interaction should be asymptotically free. We introduce an example of such a model in the next subsection. 


\begin{tabular}{|c|c|c|c|c|}
\hline & $\mathrm{SU}(2)_{X}$ & $\mathrm{SU}(2)_{W}$ & $\mathrm{U}(1)_{Y}$ & $\mathrm{SU}(3)_{c}$ \\
\hline$H_{u}$ & $\mathbf{2}$ & & $1 / 2$ & \\
$H_{d}$ & $\mathbf{2}$ & & $-1 / 2$ & \\
$S$ & $\mathbf{2}$ & $\mathbf{2}$ & & \\
$\phi_{u}$ & & $\mathbf{2}$ & $1 / 2$ & \\
$\phi_{d}$ & & $\mathbf{2}$ & $-1 / 2$ & \\
$Q_{3}$ & $\mathbf{2}$ & & $1 / 6$ & $\mathbf{3}$ \\
$Q_{1,2}$ & & $\mathbf{2}$ & $1 / 6$ & $\mathbf{3}$ \\
$\bar{u}_{1,2,3}$ & & & $-2 / 3$ & $\overline{\mathbf{3}}$ \\
$\bar{e}_{1,2,3}$ & & & 1 & \\
$\bar{d}_{1,2,3}$ & & & $1 / 3$ & $\overline{\mathbf{3}}$ \\
$L_{3}$ & $\mathbf{2}$ & & $-1 / 2$ & \\
$L_{1,2}$ & & $\mathbf{2}$ & $-1 / 2$ & \\
\hline
\end{tabular}

Table 1. The matter content of the model.

\subsection{A model with an extra SU(2) symmetry}

We borrow the setup described in ref. [15]. The gauge symmetry of the theory is $\mathrm{SU}(2)_{X} \times$ $\mathrm{SU}(2)_{W} \times \mathrm{U}(1)_{Y} \times \mathrm{SU}(3)_{c}$. The chiral multiplets are shown in table 1. Among quark and leptons, the first and the second generation doublets are charged under $\mathrm{SU}(2)_{W}$, while the third generation doublets are charged under $\mathrm{SU}(2)_{X}$. The Higgs multiplets $H_{u}$ and $H_{d}$ are charged under $\mathrm{SU}(2)_{X}$, and their vacuum expectation values (VEVs) give masses to the third generation quark/lepton. The masses of the first and the second generations are given by the VEVs of $\phi_{u}$ and $\phi_{d}$. We are interested in the case where the gauge coupling constant of $\mathrm{SU}(2)_{X}, g_{X}$, is much larger than that of $\mathrm{SU}(2)_{W}, g_{W}$ ("W" stands for "weak", and " $X$ " does for "extra"). The gauge coupling $g_{X}$ is asymptotically free.

The VEV of the bi-fundamental field $S$ breaks $\mathrm{SU}(2)_{X} \times \mathrm{SU}(2)_{W}$ down to the diagonal subgroup $\mathrm{SU}(2)_{L}$, and the low energy theory is given by the MSSM. In order to break the symmetry we consider the superpotential

$$
W=\kappa \Xi\left(S_{12} S_{21}-M^{2}\right),
$$

where $\Xi$ is a singlet chiral multiplet and $\kappa, M$ are constants, and the soft mass

$$
V_{\text {soft }}=m_{S}^{2}|S|^{2} .
$$

The VEV of $S$ is given by

$$
\langle S\rangle=\left(\begin{array}{cc}
0 & v_{S} \\
v_{S} & 0
\end{array}\right), \quad v_{S}=\sqrt{M^{2}-m^{2} / \kappa^{2}} .
$$

The gauge coupling of $\mathrm{SU}(2)_{L}$ is

$$
\frac{1}{g^{2}}=\frac{1}{g_{X}^{2}}+\frac{1}{g_{W}^{2}} \simeq \frac{1}{g_{W}^{2}} .
$$


Since in the present paper we focus on large values of $g_{X}$ in the last approximated equality we assumed $g_{X}^{2} \gg g_{W}^{2}$. The mass of the gauge boson of the broken symmetry is given by

$$
m_{X}^{2}=\left(g_{X}^{2}+g_{W}^{2}\right) v_{S}^{2}
$$

The experimental lower bound of $m_{X}$ is $m_{X} \gtrsim g_{X} \times 4.1 \mathrm{TeV}$ [32]. After integrating out the field $S$, the effective quartic coupling arising from the $\mathrm{SU}(2)_{X} \times \mathrm{SU}(2)_{W}$ gauge symmetry is given by

$$
\begin{aligned}
V_{\text {eff }} & =\left(\frac{g^{2}}{8}+\delta \lambda\right)\left(\left|H_{u}\right|^{2}-\left|H_{d}\right|^{2}\right)^{2}, \\
\delta \lambda & =\frac{\left(g_{X}^{2}-g^{2}\right) m_{S}^{2}}{4\left(m_{X}^{2}+2 m_{S}^{2}\right)} \simeq \frac{g_{X}^{2}}{8}\left(1-\epsilon^{2}\right), \quad \epsilon^{2} \equiv \frac{m_{X}^{2}}{m_{X}^{2}+2 m_{S}^{2}},
\end{aligned}
$$

In order to obtain a quartic coupling larger than that of the MSSM, the soft mass $m_{S}^{2}$ must be non-zero or, equivalently, $\epsilon^{2}<1$. The one-loop threshold correction around the symmetry breaking scale generates a soft mass of the Higgs, which in the limit $g_{X}^{2} \gg g_{W}^{2}$ is given by

$$
\delta m_{H_{u}}^{2} \simeq 3 \frac{g_{X}^{2}}{64 \pi^{2}} m_{X}^{2} \ln \left(\epsilon^{-2}\right) .
$$

This threshold correction shifts also the soft masses of all other $\mathrm{SU}(2)_{X}$-charged fields. We found that the fine-tuning of the electroweak scale from $m_{S}^{2}$ that enters via the above threshold correction is at most $10 \%$ and always subdominant as compared to the finetuning from stops and gluino, see section 3 for more details.

The Higgs mass in this model is given by

$$
m_{h}^{2}=\left(M_{Z}^{2}+4 \delta \lambda v^{2}\right) \cos ^{2}(2 \beta)+\left(\delta m_{h}^{2}\right)_{\text {loop }},
$$

where $v \approx 174 \mathrm{GeV}$ and we assumed the decoupling limit in which the mass of the heavy Higgs doublets $m_{H} \gg m_{h}$. $\left(\delta m_{h}^{2}\right)_{\text {loop }}$ parameterizes the loop correction which, similarly as in the MSSM, is dominated by loops involving stops. In the MSSM, for the stop masses of 1 (2) TeV without stop mixing the Higgs mass is only about 110 (115) GeV even for large $\tan \beta$ [37]. In the present model the $125 \mathrm{GeV}$ Higgs mass can be easily obtained for any stop mass by appropriate choice of $g_{X}, \epsilon^{2}$ and $\tan \beta$. We consider a case with a large gauge coupling of the new interaction, $g_{X} \gtrsim 2$. The $125 \mathrm{GeV}$ Higgs mass then requires values of $\epsilon^{2}$ close to unity; e.g. for the stop masses of 1 (2) TeV without stop mixing and $g_{X}=2.5$ the $125 \mathrm{GeV}$ Higgs mass requires $\epsilon^{2} \approx 0.96(0.98)$ for large $\tan \beta$. We do not consider $\tan \beta$ close to unity as it requires large stop masses and/or $\epsilon^{2}$ away from unity, which leads to fine-tuning. For a very large $\tan \beta$ close to 60 , the bottom yukawa coupling is not negligible. In particular, the quantum correction from the bottom yukawa coupling gives a negative contribution to the Higgs mass. Thus we also do not consider a very large $\tan \beta$.

The renormalization group (RG) running of the top Yukawa coupling is given by

$$
\frac{\mathrm{d}}{\mathrm{d} \ln \mu_{R}} y_{t}=\frac{y_{t}}{16 \pi^{2}}\left(-3 g_{X}^{2}-\frac{16}{3} g_{3}^{2}-\frac{13}{15} g_{1}^{2}+6 y_{t}^{2}\right) \text {. }
$$




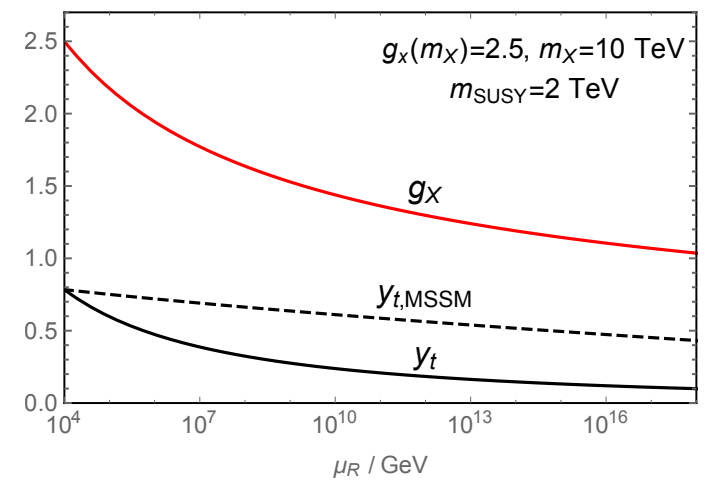

Figure 1. RG running of the gauge coupling $g_{X}$ (red) and the top Yukawa coupling $y_{t}$ for $m_{X}=$ $10 \mathrm{TeV}, m_{\mathrm{SUSY}}=2 \mathrm{TeV}, g_{X}\left(m_{X}\right)=2.5$.

In figure 1 we show the running of the gauge coupling $g_{X}$ and the top Yukawa coupling $y_{t}$. For comparison we also show the running of the top Yukawa coupling in the MSSM. We see that the Yukawa coupling is strongly suppressed as compared to the MSSM. In figure 1 we fix $g_{X}\left(m_{X}\right)=2.5$ but as we will see in section 3 , the fine-tuning measure changes only by few percent for $g_{X}$ in the range between 2 and 3 . The RG running of $g_{X}$ is slow and $g_{X}$ remains that large for a wide range of the energy scale $\mu_{R}$ before the theory exhibits strong dynamics. So there is no fine-tuning with respect to the choice of $g_{X}$ and the symmetry breaking scale. We investigate the impact of the suppressed top Yukawa coupling to the naturalness of the EWSB scale in the next section.

\section{$3 \quad$ Naturalness of electroweak symmetry breaking}

In this section we evaluate the naturalness of the EWSB scale of the model explained in the previous section. We quantify the fine-tuning using the following measure

$$
\Delta \equiv \max \left\{\Delta_{x_{i}}\right\}, \quad \Delta_{x_{i}} \equiv\left|\frac{\partial \ln v^{2}}{\partial \ln x_{i}(\Lambda)}\right|
$$

where $x_{i}$ is a mass-squared parameter (e.g. $M_{3}^{2}, m_{Q_{3}}^{2}, \mu^{2}$ ) at the mediation scale of SUSY breaking $\Lambda . \mu^{2}$ is a supersymmetric parameter and contributes to fine-tuning at tree level:

$$
\Delta_{\mu^{2}} \approx \frac{2 \mu^{2}}{m_{h}^{2}}
$$

The soft SUSY breaking parameters contribute to fine-tuning via loop corrections to $m_{H_{u}}^{2}$. In order to compute these corrections we solve the RG equations (RGEs) between the SUSY scale, set to $2 \mathrm{TeV}$, and $\Lambda$. We have set $\tan \beta=10$ and neglected all the Yukawa couplings other than the top Yukawa coupling. The RGEs are solved basically at the oneloop level except for the followings: two-loop corrections to soft masses from the first two generations of sfermions are included as we will also discuss the case where they are heavy. As $g_{X}$ is large, the RGEs of the gauge couplings are solved at the two-loop level. Two-loop corrections from the soft masses of $\mathrm{SU}(2)_{X}$-charged chiral multiplets are also included. 
Between $\Lambda$ and $m_{X}$, the RGEs are solved in the unbroken $\mathrm{SU}(2)_{X}$ phase. The size of the two-loop correction to $m_{H_{u}}^{2}$ from $m_{S}^{2}$, which for $\epsilon^{2}=0.95$ and $m_{X} \approx 10 \mathrm{TeV}$ is about $(1.5 \mathrm{TeV})^{2}$ at the $m_{X}$ scale, depends on the RG running of $m_{S}^{2}$. In our analysis we assume that $m_{S}^{2}$ is constant which is approximately the case if the $\mathrm{SU}(2)_{X}$ gaugino mass and the soft mass of $\Xi$ are suppressed. In this approximation $m_{S}^{2}$ corrects $m_{H_{u}}^{2}$ by about $-(300 \mathrm{GeV})^{2}$ for $g_{X}=2.5$ so has subdominant impact on the fine-tuning.

At a scale $m_{X}$ we perform matching by including the threshold correction (2.10) to soft masses for all $\mathrm{SU}(2)_{X}$-charged fields. The exact size of this correction depends on the parameters determining the Higgs mass. In the numerical calculations we set for concreteness $g_{X}=2.5, \epsilon^{2} \approx 0.95$ and $\tan \beta=10$ which can explain the $125 \mathrm{GeV}$ Higgs mass for sub-TeV stop masses even without stop mixing. For this choice of parameters, and after saturating the experimental lower limit on $m_{X}$ shown in the previous section, the threshold correction is about $(400 \mathrm{GeV})^{2}$ and does not affect fine-tuning much. For larger stop masses and/or non-negligible stop mixing the threshold correction can be even smaller due to $\epsilon^{2}$ being closer to unity. Between $m_{X}$ and the SUSY scale we solve the MSSM RGEs.

In order to understand how the new interaction impacts the naturalness of EWSB it is instructive to express the IR value of $m_{H_{u}}^{2}$ in terms of the most relevant UV soft SUSY breaking parameters. For the MSSM RGEs, which is a useful reference point, this relation is given by

$$
\begin{aligned}
\left(m_{H_{u}}^{2}\right)_{\mathrm{IR}}^{\mathrm{MSSM}} \approx & 0.68 m_{H_{u}}^{2}-0.32 m_{Q_{3}}^{2}-0.25 m_{U_{3}}^{2}+0.005 m_{0}(1,2)^{2} \\
& -1.37 M_{3}^{2}+0.21 M_{2}^{2}-0.13 M_{2} M_{3},
\end{aligned}
$$

where all soft terms on the r.h.s. are defined at the UV scale $\Lambda=10^{16} \mathrm{GeV} \cdot m_{0}(1,2)^{2}$ is the soft masses of the first two generation sfermions. The above formula clearly demonstrates the well-known fact that fine-tuning in the MSSM is dominated by stops and gluino which all give negative contribution to $m_{H_{u}}^{2}$ via the renormalization by the large top Yukawa coupling.

In the presence of a large new gauge coupling the top Yukawa coupling is driven to smaller values at high energy scales. In the model considered in this paper with $g_{X}=2.5$ the relation between the IR value of $m_{H_{u}}^{2}$ and the UV parameters reads:

$$
\begin{aligned}
\left(m_{H_{u}}^{2}\right)_{\mathrm{IR}} \approx & 0.82 m_{H_{u}}^{2}-0.22 m_{Q_{3}}^{2}-0.08 m_{U_{3}}^{2}+0.005 m_{0}(1,2)^{2} \\
& -1.1 M_{3}^{2}+8.9 M_{X}^{2}-1.39 M_{X} M_{3},
\end{aligned}
$$

where $M_{X}$ is the $\mathrm{SU}(2)_{X}$ gaugino mass. Note that the coefficient in front of $m_{U_{3}}^{2}$ is reduced by a factor of three as compared to the MSSM case, as a consequence of suppressed top Yukawa coupling at high energy scales. The coefficients in front of $m_{Q_{3}}^{2}$ and $M_{3}^{2}$ are also reduced but not as much. This is due to compensating effects induced by large $g_{X}$. Namely, $m_{Q_{3}}^{2}$ gives additional negative two-loop correction to $m_{H_{u}}^{2}$ by the $\mathrm{SU}(2)_{X}$ gauge interaction,

$$
\frac{\mathrm{d}}{\mathrm{d} \ln \mu_{R}} m_{H_{u}}^{2}=\frac{3 g_{X}^{4}}{256 \pi^{4}} \times 3 m_{Q_{3}}^{2}+\cdots
$$


while $M_{3}$ gives a negative contribution to $m_{H_{u}}^{2}$ via the Higgs-stop trilinear coupling $A_{t}$,

$$
\begin{aligned}
\frac{\mathrm{d}}{\mathrm{d} \ln \mu_{R}} m_{H_{u}}^{2} & =\frac{6 y_{t}^{2}}{16 \pi^{2}} A_{t}^{2}+\cdots, \\
\frac{\mathrm{d}}{\operatorname{dln} \mu_{R}} A_{t} & =\frac{A_{t}}{16 \pi^{2}}\left(18 y_{t}^{2}-\frac{16}{3} g_{3}^{2}-3 g_{X}^{2}\right)+\frac{g_{3}^{2}}{16 \pi^{2}} \frac{32}{3} M_{3}+\cdots
\end{aligned}
$$

The effect of $g_{X}$ dominates one-loop RG running of the top trilinear coupling $A_{t}$ so $A_{t}=0$ is no longer an attractive solution (as it is the case in the MSSM [38]). This amplifies $\left|A_{t}\right|$ which is renormalized by the gluino mass and substantially feeds into the $\beta$-function of $m_{H_{u}}^{2}$ via the top Yukawa coupling (despite the latter being suppressed). As a consequence of these compensating effects of $y_{t}$ and $g_{X}$ the coefficients in front of $m_{Q_{3}}^{2}$ and $M_{3}^{2}$ are stable as a function of $g_{X}$ and vary by only few percent for $g_{X}$ in the range between 2 an 3 . We set $g_{X}=2.5$ in the rest of our numerical analysis of the present paper. We also see from eq. (3.4) a large dependence of $m_{H_{u}}^{2}$ on the $\mathrm{SU}(2)_{X}$ gaugino mass $M_{X}$ so we set it to zero throughout the analysis.

Fine-tuning in various supersymmetric extensions of the SM has been usually computed assuming some correlations between the UV parameters, see e.g. [39-49]. In the present paper we remain agnostic about any such correlations and take purely phenomenological point of view and calculate the fine-tuning measure as a function of physical masses of sparticles. In this approach collider constraints on sparticle masses may be translated to lower bounds on $\Delta$. Such procedure was recently adopted in ref. [24] using the MSSM RGEs. However, in the work of ref. [24] $m_{H_{u}}^{2}$ was not included in the set of parameters $\left\{x_{i}\right\}$ for which the fine-tuning is calculated. We think that $\Delta_{m_{H_{u}}^{2}}$ should be included in the definition of $\Delta$ and do it accordingly. In fact, $\Delta_{m_{H_{u}}^{2}}$ often dominates the total amount of fine-tuning unless there is some large positive contribution to $\left(m_{H_{u}}^{2}\right)_{\mathrm{IR}}$ that approximately cancels that from gluino and stops which allows for a small value of $m_{H_{u}}^{2}$ at the mediation scale.

In the left panel of figure 2 we present the fine-tuning as a function of the IR soft gluino mass and the mass of stops for $\mu=M_{1}=M_{2}=200 \mathrm{GeV}$. This corresponds to a generic case in which the negative corrections from stops and gluino are cancelled by a non-negligible UV value of $m_{H_{u}}^{2}$ and $\Delta_{m_{H_{u}}^{2}}$ tends to dominate the fine-tuning. We see that for a given value of $\Delta$, gluino and stop masses can be heavier than in the MSSM by several hundred $\mathrm{GeV}$. The tuning better than $1 \%$ can be obtained for a gluino mass up to $2 \mathrm{TeV}$ and stop masses up to $1.5 \mathrm{TeV}$, as compared to 1.8 and $1.2 \mathrm{TeV}$ in the MSSM, respectively. The biggest improvement in tuning is in the region $m_{\text {stop }} \gg M_{3}$ where corrections from stops dominate tuning and $\Delta$ is almost a factor three smaller than in the MSSM.

We also note that the improvement is particularly significant in a well-motivated scenario in which the first two generation of sfermions are much heavier than the third one. This scenario was suggested as a way to ease SUSY flavor problems [50-55]. However, it was pointed out that two-loop RGEs from heavy 1st/2nd generation of sfermions lead to tachyonic stops unless the latter have a large soft terms at the UV scale in tension with naturalness [56]. The size of this effect can be understood by expressing the stop masses 

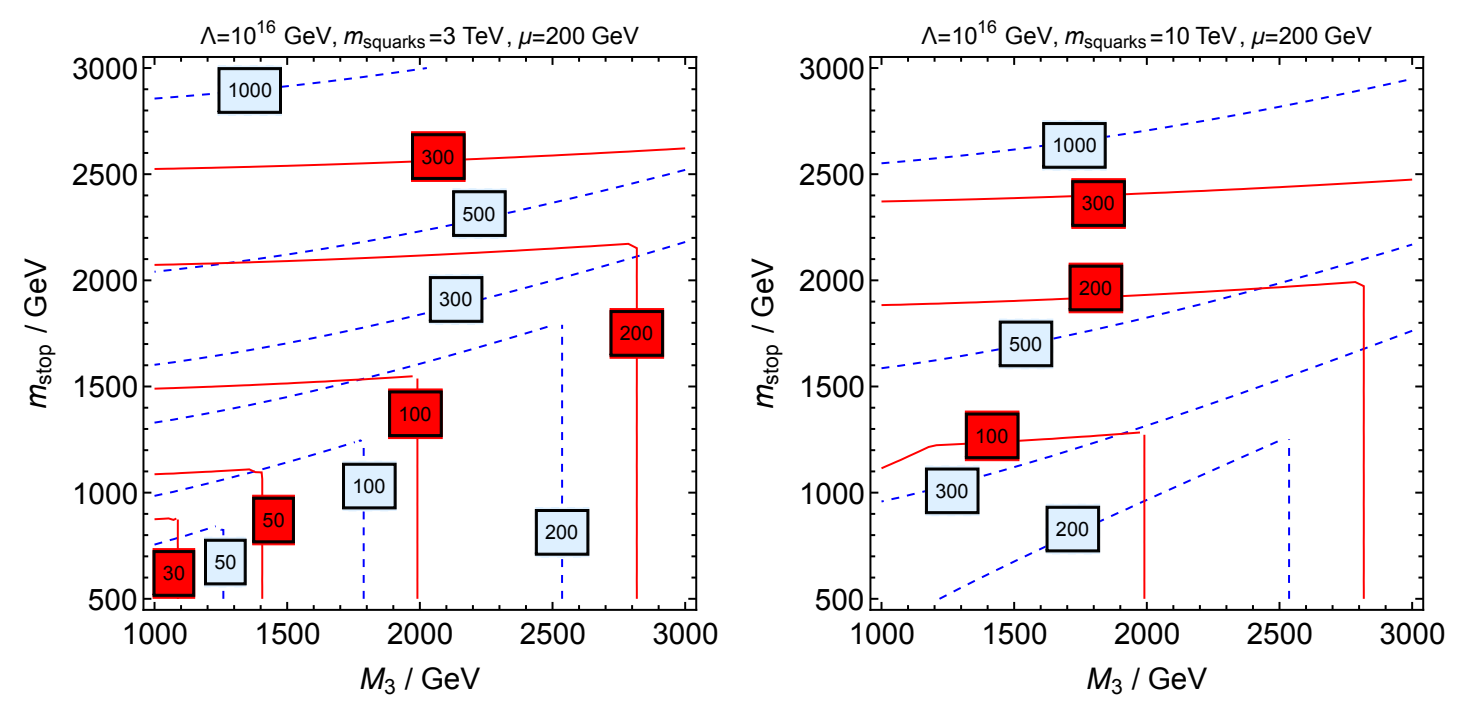

Figure 2. Contours of the fine-tuning measure $\Delta$ in the plane of IR values of the soft gluino mass $M_{3}$ and the mass of degenerate stops $m_{\text {stop }}$. The solid red contours corresponds to $g_{X}=2.5$, $\epsilon^{2}=0.95$ and $\tan \beta=10$. Values of $\Delta$ in MSSM are depicted by the blue dashed contours for comparison. In the left (right) panel the sfermions of the first two generations are set to 3 (10) $\mathrm{TeV}$. The remaining parameters are set to values which do not lead to substantial corrections to the EWSB scale: $\mu=M_{1}=M_{2}=200 \mathrm{GeV}$ and the soft $\mathrm{SU}(2)_{X}$ gaugino mass $M_{X}=0$.

in IR as a function of the UV soft terms;

$$
\begin{gathered}
\left(m_{Q_{3}}^{2}\right)_{\mathrm{IR}}^{\mathrm{MSSM}} \approx-0.11 m_{H_{u}}^{2}+0.89 m_{Q_{3}}^{2}-0.08 m_{U_{3}}^{2}-0.03 m_{0}(1,2)^{2} \\
\quad 3.4 M_{3}^{2}+0.33 M_{2}^{2}-0.04 M_{2} M_{3} \\
\left(m_{U_{3}}^{2}\right)_{\mathrm{IR}}^{\mathrm{MSSM}} \approx-0.16 m_{H_{u}}^{2}-0.17 m_{Q_{3}}^{2}+0.73 m_{U_{3}}^{2}-0.02 m_{0}(1,2)^{2} \\
2.9 M_{3}^{2}-0.12 M_{2}^{2}-0.08 M_{2} M_{3}
\end{gathered}
$$

in the MSSM and

$$
\begin{gathered}
\left(m_{Q_{3}}^{2}\right)_{\mathrm{IR}} \approx-0.07 m_{H_{u}}^{2}+0.87 m_{Q_{3}}^{2}-0.03 m_{U_{3}}^{2}-0.02 m_{0}(1,2)^{2} \\
\quad 3.4 M_{3}^{2}+11.3 M_{X}^{2}-0.45 M_{X} M_{3} \\
\left(m_{U_{3}}^{2}\right)_{\mathrm{IR}} \approx-0.06 m_{H_{u}}^{2}-0.05 m_{Q_{3}}^{2}+0.84 m_{U_{3}}^{2}-0.02 m_{0}(1,2)^{2} \\
\quad 2.9 M_{3}^{2}-2.5 M_{X}^{2}-0.93 M_{X} M_{3}
\end{gathered}
$$

in the model with the new gauge interaction. We see that at one-loop level stops are mainly renormalized by the gluino mass. However, for the mass of the 1st/2nd generation of sfermions of $\mathcal{O}(10) \mathrm{TeV}$ the 2-loop effect may dominate. Notice also that this 2-loop effect in the stops RGEs feeds into the RG running of $m_{H_{u}}^{2}$. This is the reason for the positive coefficients in front of $m_{0}(1,2)^{2}$ in eqs. (3.3)-(3.4) and it may result in destabilization of the EW scale if the 1st/2nd generation of sfermions are too heavy [57].

In the right panel of figure 2 we show contours of $\Delta$ for heavy first two generations of sfermions with their masses set to $10 \mathrm{TeV}$. We see that fine-tuning in the MSSM is always 

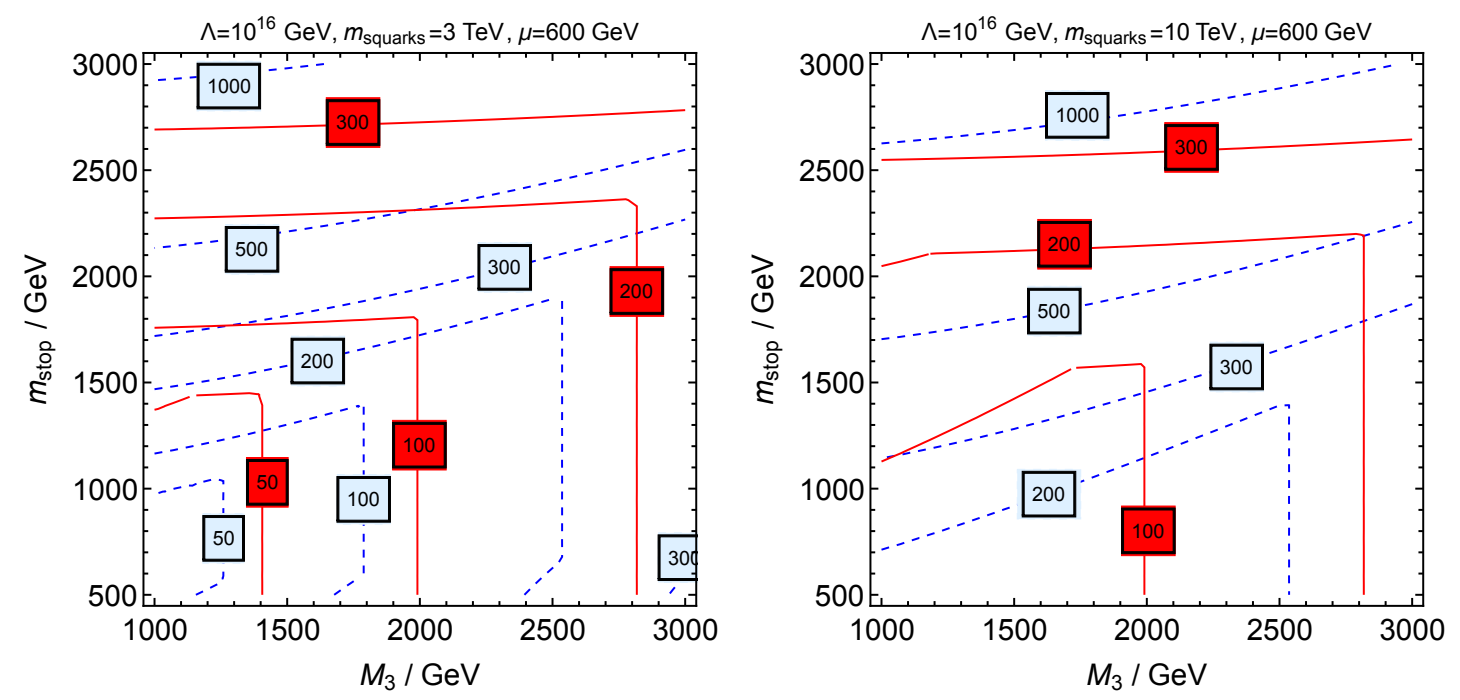

Figure 3. The same as in figure 2 but for $\mu=600 \mathrm{GeV}$.

worse than $1 \%$. The reason is that even for light stops the soft masses of stops must be large at the mediation scale to compensate for the negative contribution from the heavy first two generation. This is true to large extent also for the model with the new gauge interaction ${ }^{1}$ but the sensitivity of the EW scale to the soft stop masses is smaller due to the suppressed top Yukawa coupling. In consequence, in the model with the new gauge interaction the region with tuning better than $1 \%$ is only mildly affected by shifting the maximal value of the stop masses from 1.5 to $1.3 \mathrm{TeV}$. We should also note that the threshold corrections to the stop masses from the heavy first two generation of squarks, which we neglected in our analysis, are generically large and positive [58]. For the $10 \mathrm{TeV}$ squarks the physical stop masses may be about $20 \%$ larger than their tree-level value, as was recently emphasized in ref. [23]. After taking this into account, the range of stop masses with the tuning better than $1 \%$ is essentially unaffected by heavy 1 st/2nd generation of sfermions.

The parameters in figure 2 were chosen in such a way that $m_{H_{u}}^{2}$ is not suppressed at the UV scale so $\Delta_{m_{H_{u}}^{2}}$ dominates the total fine-tuning over much of the parameter space. $m_{H_{u}}^{2}$ at the UV scale can be decreased by increasing $|\mu|$ and/or any of the soft terms on the r.h.s. of eq. (3.4) with positive coefficients. In figure 3 contours of $\Delta$ are shown for $\mu=600 \mathrm{GeV}$. For such value of $\mu, \Delta_{\mu^{2}}$ is below 50 so does not affect $\Delta$ (except for gluino mass close to $1 \mathrm{TeV}$ which is excluded anyway for typical SUSY spectra). We see that this choice improves the fine-tuning by a factor of two in comparison with $\mu=200 \mathrm{GeV}$ in some of the parameter space and shifts the upper bound on the stop masses with the tuning better than $1 \%$ up to 1.8 (1.6) $\mathrm{TeV}$ for the mass of the first two generation of sfermions set to 3 (10) TeV. Similar effect on the fine-tuning is also present in the MSSM so the tuning is still improved by up to a factor of three.

We have neglected so far the impact of the Higgs mass constraint in the comparison of the fine-tuning of the present model to the MSSM. In our analysis we assumed vanishing $A_{t}$

\footnotetext{
${ }^{1}$ Notice also that the coefficient in eq. (3.10) in front of $m_{0}(1,2)^{2}$ is smaller than in eq. (3.8) for the MSSM which is due to the absence of the two-loop renormalization by the $\mathrm{SU}(2)_{L}$ gauge coupling for $m_{Q_{3}}^{2}$. For a given stop mass this results in smaller UV value of $m_{Q_{3}}^{2}$ than in the MSSM, hence smaller tuning.
} 
at the UV scale which generically leads to negligible stop mixing. In the MSSM with vanishing stop mixing the $125 \mathrm{GeV}$ Higgs mass implies the stop masses of at least $10 \mathrm{TeV}$ [37] which leads to $\Delta \gtrsim 10^{4}$. The mass of stops required to obtain the correct Higgs mass in the MSSM can be reduced to about $2 \mathrm{TeV}$ if the contribution to the Higgs mass from the stop mixing is maximized which occurs for $\left|A_{t}\right| \approx \sqrt{6} m_{\text {stop }}$ at the SUSY scale. The maximal stop mixing is possible even for $A_{t}=0$ at the UV scale if $A_{t}$ is driven to large negative value via strong $R G$ effects from heavy gluino while stops are kept relatively light due to heavy first two generations of sfermions [57]. This effect can be illustrated by expressing the IR value of $A_{t}$ in terms of the UV value of itself and gluino mass in the MSSM:

$$
\left(A_{t}\right)_{\mathrm{IR}}^{\mathrm{MSSM}} \approx-1.5 M_{3}+0.3 A_{t} .
$$

However, in such a scenario $2 \mathrm{TeV}$ stops with $A_{t} \approx-\sqrt{6} m_{\text {stop }}$ require gluino mass of about $7 \mathrm{TeV}$ which leads to $\Delta \approx 1500$. Switching on negative $A_{t}$ at the UV scale does not help much in reducing $\Delta$ since it has relatively small effect on its IR value while it gives large correction to $m_{H_{u}}^{2}$ via the top Yukawa coupling and easiely dominates tuning if it is large. Thus, in any case $\Delta$ in the MSSM is at least 1000 once the Higgs mass constraint is taken into account. We conclude that the model with the extra gauge interaction improves naturalness by one to two orders of magnitude as compared to the MSSM.

The fine-tuning depends somewhat on explicit implementations of the model that introduces an extra gauge interaction. If the right-handed top is charged under new gauge group instead of the left-handed one, as it is the case e.g. in a model proposed in ref. [32], we expect that fine-tuning would be further improved. This is because in such a case the two-loop correction does not affect $m_{Q_{3}}^{2}$ which dominates the tuning from stops in the MSSM and in the present model. The fine-tuning could be also affected by a change in the number of flavors charged under the extra gauge group and the rank of that gauge group.

\section{Summary and discussion}

We investigated the impact of an extra gauge interaction on the fine-tuning of the EW scale in supersymmetry. We found that if the new gauge coupling is large the fine-tuning from stops and gluino is reduced due to suppression of the top Yukawa coupling at higher scales. This effect is present in any model in which the Higgs and the top quark are charged under the new gauge symmetry and the extra gauge coupling is large.

We quantified the fine-tuning in an explicit model with $\mathrm{SU}(2)_{X} \times \mathrm{SU}(2)_{W}$ gauge symmetry which is broken to the $\mathrm{SM} \mathrm{SU}(2)_{L}$ gauge group at a scale of around $10 \mathrm{TeV}$. We found that for the mediation scale of SUSY breaking of $10^{16} \mathrm{GeV}$ the upper bound on the gluino mass from naturalness is increased by few hundred $\mathrm{GeV}$, as compared to the MSSM, e.g. for tuning better than $1 \%$ the maximal value of the gluino mass shifts from 1.8 to $2 \mathrm{TeV}$. Stops up to about $2 \mathrm{TeV}$ may also have tuning better than $1 \%$. For stops the improvement is more significant, especially in the scenario with the first two generations of sfermions much heavier than stops, and the fine-tuning may be a factor of three smaller than in the MSSM with the same stop and gluino masses. Morevoer, once the Higgs mass 
constraint is taken into account the fine-tuning in the present model is one to two orders of magnitude smaller than in the MSSM.

Our findings also demonstrate that it is not possible to find model-independent bounds on stop and gluino masses from naturalness consideration (even if one sticks to one finetuning measure) and in particular, the mechanism that raises the tree-level Higgs mass above that predicted in the MSSM may have non-negligible impact on the fine-tuning.

\section{Acknowledgments}

MB would like to thank the Berkeley Center of Theoretical Physics for its hospitality during completion of this work. This work has been partially supported by National Science Centre, Poland, under research grants no. 2017/26/D/ST2/00225 and DEC2014/15/B/ST2/02157 (MB), by the Office of High Energy Physics of the U.S. Department of Energy under Contract DE-AC02-05CH11231 (KH), and by the National Science Foundation under grant PHY-1316783 (KH).

Open Access. This article is distributed under the terms of the Creative Commons Attribution License (CC-BY 4.0), which permits any use, distribution and reproduction in any medium, provided the original author(s) and source are credited.

\section{References}

[1] L. Maiani, All you need to know about the Higgs boson, in the proceedings of the Summer School on Particle Physics, September 3-7, Paris, France (1979).

[2] M.J.G. Veltman, The infrared-ultraviolet connection, Acta Phys. Polon. B 12 (1981) 437 [INSPIRE].

[3] E. Witten, Dynamical breaking of supersymmetry, Nucl. Phys. B 188 (1981) 513 [InSPIRE].

[4] R.K. Kaul, Gauge hierarchy in a supersymmetric model, Phys. Lett. 109B (1982) 19 [INSPIRE].

[5] ATLAS, CMS collaboration, Combined measurement of the Higgs boson mass in pp collisions at $\sqrt{s}=7$ and $8 \mathrm{TeV}$ with the ATLAS and CMS experiments, Phys. Rev. Lett. 114 (2015) 191803 [arXiv: 1503.07589] [INSPIRE].

[6] U. Ellwanger, C. Hugonie and A.M. Teixeira, The next-to-minimal supersymmetric standard model, Phys. Rept. 496 (2010) 1 [arXiv:0910.1785] [INSPIRE].

[7] U. Ellwanger, A Higgs boson near $125 \mathrm{GeV}$ with enhanced di-photon signal in the NMSSM, JHEP 03 (2012) 044 [arXiv:1112.3548] [INSPIRE].

[8] L.J. Hall, D. Pinner and J.T. Ruderman, A natural SUSY Higgs near 126 GeV, JHEP 04 (2012) 131 [arXiv:1112.2703] [INSPIRE].

[9] J.-J. Cao et al., A SM-like Higgs near $125 \mathrm{GeV}$ in low energy SUSY: a comparative study for MSSM and NMSSM, JHEP 03 (2012) 086 [arXiv: 1202.5821] [INSPIRE].

[10] K.S. Jeong, Y. Shoji and M. Yamaguchi, Singlet-doublet Higgs mixing and its implications on the Higgs mass in the PQ-NMSSM, JHEP 09 (2012) 007 [arXiv:1205.2486] [INSPIRE]. 
[11] K. Agashe, Y. Cui and R. Franceschini, Natural islands for a $125 \mathrm{GeV}$ Higgs in the scale-invariant NMSSM, JHEP 02 (2013) 031 [arXiv:1209.2115] [INSPIRE].

[12] S.F. King, M. Mühlleitner, R. Nevzorov and K. Walz, Natural NMSSM Higgs Bosons, Nucl. Phys. B 870 (2013) 323 [arXiv:1211.5074] [inSPIRE].

[13] T. Gherghetta, B. von Harling, A.D. Medina and M.A. Schmidt, The scale-invariant nmssm and the $126 \mathrm{GeV}$ Higgs boson, JHEP 02 (2013) 032 [arXiv:1212.5243] [INSPIRE].

[14] M. Badziak, M. Olechowski and S. Pokorski, New regions in the NMSSM with a $125 \mathrm{GeV}$ Higgs, JHEP 06 (2013) 043 [arXiv:1304.5437] [INSPIRE].

[15] P. Batra, A. Delgado, D.E. Kaplan and T.M.P. Tait, The Higgs mass bound in gauge extensions of the minimal supersymmetric standard model, JHEP 02 (2004) 043 [hep-ph/0309149] [INSPIRE].

[16] M. Endo et al., Higgs mass and muon anomalous magnetic moment in the $\mathrm{U}(1)$ extended MSSM, Phys. Rev. D 85 (2012) 095006 [arXiv:1112.6412] [INSPIRE].

[17] C. Cheung and H.L. Roberts, Higgs mass from D-terms: a Litmus test, JHEP 12 (2013) 018 [arXiv: 1207.0234] [INSPIRE].

[18] R. Huo, G. Lee, A.M. Thalapillil and C.E.M. Wagner, $\mathrm{SU}(2) \otimes \mathrm{SU}(2)$ gauge extensions of the MSSM revisited, Phys. Rev. D 87 (2013) 055011 [arXiv:1212.0560] [INSPIRE].

[19] R.T. D'Agnolo, E. Kuflik and M. Zanetti, Fitting the Higgs to natural SUSY, JHEP 03 (2013) 043 [arXiv: 1212.1165] [INSPIRE].

[20] N. Craig and A. Katz, A supersymmetric Higgs sector with chiral D-terms, JHEP 05 (2013) 015 [arXiv: 1212.2635] [INSPIRE].

[21] E. Bertuzzo and C. Frugiuele, Natural SM-like 126 GeV Higgs boson via nondecoupling D terms, Phys. Rev. D 93 (2016) 035019 [arXiv:1412.2765] [InSPIRE].

[22] R.M. Capdevilla, A. Delgado and A. Martin, Light stops in a minimal U(1)x extension of the MSSM, Phys. Rev. D 92 (2015) 115020 [arXiv:1509.02472] [InSPIRE].

[23] M.R. Buckley et al., Cornering natural SUSY at LHC run II and beyond, JHEP 08 (2017) 115 [arXiv: 1610.08059] [INSPIRE].

[24] M.R. Buckley, A. Monteux and D. Shih, Precision corrections to fine tuning in SUSY, JHEP 06 (2017) 103 [arXiv:1611.05873] [INSPIRE].

[25] S. Dimopoulos, K. Howe and J. March-Russell, Maximally natural supersymmetry, Phys. Rev. Lett. 113 (2014) 111802 [arXiv:1404.7554] [inSPIRE].

[26] I. Garcia Garcia, K. Howe and J. March-Russell, Natural Scherk-Schwarz theories of the weak scale, JHEP 12 (2015) 005 [arXiv: 1510.07045] [INSPIRE].

[27] P.J. Fox, A.E. Nelson and N. Weiner, Dirac gaugino masses and supersoft supersymmetry breaking, JHEP 08 (2002) 035 [hep-ph/0206096] [INSPIRE].

[28] A. Arvanitaki et al., The last vestiges of naturalness, JHEP 03 (2014) 022 [arXiv: 1309.3568] [INSPIRE].

[29] N. Craig and K. Howe, Doubling down on naturalness with a supersymmetric twin Higgs, JHEP 03 (2014) 140 [arXiv: 1312.1341] [INSPIRE].

[30] A. Katz et al., SUSY meets her twin, JHEP 01 (2017) 142 [arXiv:1611.08615] [INSPIRE]. 
[31] M. Badziak and K. Harigaya, Supersymmetric D-term twin higgs, JHEP 06 (2017) 065 [arXiv: 1703.02122] [INSPIRE].

[32] M. Badziak and K. Harigaya, Minimal non-abelian supersymmetric twin Higgs, JHEP 10 (2017) 109 [arXiv: 1707.09071] [INSPIRE].

[33] M. Badziak and K. Harigaya, Asymptotically free natural supersymmetric twin Higgs model, Phys. Rev. Lett. 120 (2018) 211803 [arXiv:1711.11040] [INSPIRE].

[34] Z. Chacko, H.-S. Goh and R. Harnik, The twin Higgs: natural electroweak breaking from mirror symmetry, Phys. Rev. Lett. 96 (2006) 231802 [hep-ph/0506256] [INSPIRE].

[35] A. Falkowski, S. Pokorski and M. Schmaltz, Twin SUSY, Phys. Rev. D 74 (2006) 035003 [hep-ph/0604066] [INSPIRE].

[36] S. Chang, L.J. Hall and N. Weiner, A supersymmetric twin Higgs, Phys. Rev. D 75 (2007) 035009 [hep-ph/0604076] [INSPIRE].

[37] J. Pardo Vega and G. Villadoro, SusyHD: Higgs mass determination in supersymmetry, JHEP 07 (2015) 159 [arXiv:1504.05200] [INSPIRE].

[38] M. Carena et al., Radiative electroweak symmetry breaking and the infrared fixed point of the top quark mass, Nucl. Phys. B 419 (1994) 213 [hep-ph/9311222] [INSPIRE].

[39] P.H. Chankowski, J.R. Ellis and S. Pokorski, The fine tuning price of LEP, Phys. Lett. B 423 (1998) 327 [hep-ph/9712234] [INSPIRE].

[40] J.L. Feng, K.T. Matchev and T. Moroi, Multi-TeV scalars are natural in minimal supergravity, Phys. Rev. Lett. 84 (2000) 2322 [hep-ph/9908309] [INSPIRE].

[41] H. Abe, T. Kobayashi and Y. Omura, Relaxed fine-tuning in models with non-universal gaugino masses, Phys. Rev. D 76 (2007) 015002 [hep-ph/0703044] [INSPIRE].

[42] D. Horton and G.G. Ross, Naturalness and Focus Points with Non-Universal Gaugino Masses, Nucl. Phys. B 830 (2010) 221 [arXiv:0908.0857] [INSPIRE].

[43] G.G. Ross and K. Schmidt-Hoberg, The fine-tuning of the generalised NMSSM, Nucl. Phys. B 862 (2012) 710 [arXiv:1108.1284] [InSPIRE].

[44] T.T. Yanagida and N. Yokozaki, Focus point in gaugino mediation $\sim$ reconsideration of the fine-tuning problem, Phys. Lett. B 722 (2013) 355 [arXiv:1301.1137] [INSPIRE].

[45] A. Kaminska, G.G. Ross and K. Schmidt-Hoberg, Non-universal gaugino masses and fine tuning implications for SUSY searches in the MSSM and the GNMSSM, JHEP 11 (2013) 209 [arXiv: 1308.4168] [INSPIRE].

[46] K. Kowalska, L. Roszkowski, E.M. Sessolo and S. Trojanowski, Low fine tuning in the MSSM with higgsino dark matter and unification constraints, JHEP 04 (2014) 166 [arXiv: 1402.1328] [INSPIRE].

[47] K. Harigaya, T.T. Yanagida and N. Yokozaki, Seminatural SUSY from the $E_{7}$ nonlinear $\sigma$-model, PTEP 2015 (2015) 083B03 [arXiv: 1504.02266] [INSPIRE].

[48] K. Harigaya, T.T. Yanagida and N. Yokozaki, Muon g- 2 in focus point SUSY, Phys. Rev. D 92 (2015) 035011 [arXiv: 1505.01987] [INSPIRE].

[49] G.G. Ross, K. Schmidt-Hoberg and F. Staub, Revisiting fine-tuning in the MSSM, JHEP 03 (2017) 021 [arXiv:1701.03480] [INSPIRE]. 
[50] M. Dine, R.G. Leigh and A. Kagan, Flavor symmetries and the problem of squark degeneracy, Phys. Rev. D 48 (1993) 4269 [hep-ph/9304299] [InSPIRE].

[51] A. Pomarol and D. Tommasini, Horizontal symmetries for the supersymmetric flavor problem, Nucl. Phys. B 466 (1996) 3 [hep-ph/9507462] [INSPIRE].

[52] E. Dudas, S. Pokorski and C.A. Savoy, Soft scalar masses in supergravity with horizontal U(1)x gauge symmetry, Phys. Lett. B 369 (1996) 255 [hep-ph/9509410] [INSPIRE].

[53] R. Barbieri, G.R. Dvali and L.J. Hall, Predictions from a U(2) flavor symmetry in supersymmetric theories, Phys. Lett. B 377 (1996) 76 [hep-ph/9512388] [INSPIRE].

[54] E. Dudas, C. Grojean, S. Pokorski and C.A. Savoy, Abelian flavor symmetries in supersymmetric models, Nucl. Phys. B 481 (1996) 85 [hep-ph/9606383] [INSPIRE].

[55] A.G. Cohen, D.B. Kaplan and A.E. Nelson, The more minimal supersymmetric standard model, Phys. Lett. B 388 (1996) 588 [hep-ph/9607394] [INSPIRE].

[56] N. Arkani-Hamed and H. Murayama, Can the supersymmetric flavor problem decouple?, Phys. Rev. D 56 (1997) R6733 [hep-ph/9703259] [InSPIRE].

[57] M. Badziak et al., Inverted sfermion mass hierarchy and the Higgs boson mass in the MSSM, JHEP 07 (2012) 155 [arXiv: 1205.1675] [INSPIRE].

[58] D.M. Pierce et al., Precision corrections in the minimal supersymmetric standard model, Nucl. Phys. B 491 (1997) 3 [hep-ph/9606211] [INSPIRE]. 